\title{
Uso de un recurso educativo digital en la enseñanza-aprendizaje de la inserción de catéter venoso periférico
}

\section{Use of a digital educational resource in teaching - learning insertion of a peripheral venous catheter}

Consuelo Ortiz Suarez ${ }^{10}$, Ximena Sáenz Montoya ${ }^{20}$, Juan Carlos Diaz Alvarez ${ }^{3}$, Luz Nelly Rivera Alvarez ${ }^{4}{ }^{*}$

${ }^{1}$ Universidad Nacional de Colombia. Bogotá, Colombia.

*Dirigir correspondencia a: cortizs@unal.edu.co

\begin{tabular}{l}
\hline \\
\hline Proceso Editorial \\
\hline Recibido: 14122020 \\
Aceptado: 16072021 \\
Publicado:10 022022 \\
\hline
\end{tabular}

DOI 10.17081/innosa.144

(c) Copyright 2022.

Ortiz ${ }^{1}$ et al.

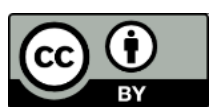

\section{RESUMEN}

Introducción: Las tecnologías de la información y la comunicación combinadas con simulación clínica optimizan los procesos de enseñanza - aprendizaje en la formación del recurso humano en salud. Métodos: estudio cuasiexperimental en 91 participantes asignados en tres grupos. El G1 recibió la intervención 1, el G2 recibió la intervención 2 y el grupo control recibió la intervención tradicional. El conocimiento teórico-conceptual se midió con una prueba de conocimiento y la habilidad procedimental con una lista de chequeo. Resultados: el aprendizaje teórico-conceptual y la habilidad procedimental mostró diferencias significativas entre los grupos $(p=0.01)$, con mayores puntajes en los grupos de intervención frente al GC que; aunque se presentó un ligero incremento en los puntajes, este no superó los G1 y G2; y Conclusiones: La enseñanza aprendizaje con el uso del RED "inserción del catéter venoso periférico" es eficaz, porque incrementa el aprendizaje teórico, conceptual y de habilidad procedimental en estudiantes de enfermería y es significativamente mejor cuando este se acompaña de un docente adherido al contenido del recurso. EI RED en simulación favorece el aprendizaje autónomo, y se constituye en una herramienta útil, en la formación de pregrado, y en programas de educación continua.

Palabras clave: entrenamiento de simulación; cateterismo periférico; educación en enfermería; sistema virtual; aprendizaje.

\begin{abstract}
Background: The use of information and communication technologies combined with clinical simulation, optimize the teaching- learning processes in the training of health care human resource. Objective: evaluate the effectiveness of the digital resource (RED) "insertion of a peripheral venous catheter" in the theoretical, conceptual, and procedural learning of nursing students. Place the question addressed in a broad context and highlight the purpose of the study. Methods: quasi-experimental study under carried within 91 participants separated into three learning groups. Group 1 was trained in intervention 1; Group 2 in intervention 2, and the Control Group CG received a traditional intervention scheme. The theoretical-conceptual knowledge was measured throughout a test and the procedural skills were measured thoughout a checklist. Results: theoretical-conceptual learning and procedural skills showed significant differences between the groups $(p=0.01)$. Intervention groups achieved higher scores when compared to the CG, for which although there was a slight increase in scores, these did not exceed the ones achieved by G1 and G2. Conclusions: The teaching-learning with the use of the RED "insertion of the peripheral venous catheter" is effective, because it increases theoretical, conceptual and procedural skill learning in nursing students and is significantly better when it is accompanied by a teacher adhering to the content of the resource. The RED in simulation favors autonomous learning, and constitutes a useful tool in undergraduate training and in continuing education programs.
\end{abstract}

Keywords: simulation training; catheterization peripheral; nursing education; virtual system; learning. 


\section{INTRODUCCIÓN}

La inserción del catéter venoso periférico es un procedimiento clínico utilizado con mucha frecuencia en la atención en salud $(1,2)$ y es una práctica habitual de enfermería que no la exime de eventos adversos y complicaciones (므). Las estrategias de enseñanza se han dado en los programas de formación mediante clases teóricas en el aula, demostraciones, cursos de elearning, videos y práctica en simuladores de miembros superiores y dispositivos hápticos (2), sin embargo, el uso de estrategias mixtas, es decir, la integración de la enseñanza en línea con la enseñanza presencial, parece ser mejor en la adquisición de habilidades clínicas en estudiantes del área de la salud. El conocimiento, la confianza y las habilidades de inserción de catéteres venosos, mejoran significativamente cuando se combina el aprendizaje en línea con simulación (4).

Las características de las nuevas generaciones en la educación superior, el número creciente de estudiantes por grupo para aprender un procedimiento clínico, la variabilidad de conceptos y técnicas en los docentes que enseñan el procedimiento, así como la necesidad creciente de un recurso en salud hábil para la interacción humana y la imperiosa necesidad de garantizar un cuidado seguro en las prácticas clínicas, demandan repensar y utilizar nuevas estrategias educativas que generen mayor interés y produzcan mejores resultados en el aprendizaje.

La sociedad del conocimiento ha generado transformaciones en la educación ( $\underline{5}$ ) y ha incorporado las nuevas tecnologías de la información y las comunicaciones (TIC), las cuales se han convertido en un importante y moderno recurso en la educación superior que busca dar respuesta a las demandas académicas. Las TIC favorecen el desarrollo de prácticas educativas más pertinentes, eficaces y útiles para diversificar los procesos de enseñanza-aprendizaje (6). Así mismo, mediante estas tecnologías, se construyen contenidos con propósitos educativos, conocidos como recursos educativos digitales (RED), los cuales pueden alojarse en internet o en plataformas de aprendizaje y que por sus características suelen ser eficaces en la enseñanzaaprendizaje de procedimientos clínicos.

Los RED ofrecen un contenido teórico presentado mediante diversos recursos didácticos como como ilustraciones, audios, videos que facilitan el aprendizaje en estudiantes y se constituyen en una herramienta que facilita el desarrollo de habilidades procedimentales en escenarios de simulación cuando el aprendiz puede realizar "paso a paso" la técnica mientras la va observando en el RED en línea. La simulación permite practicar habilidades de cuidado como la inserción de catéteres y habilidades relacionales (7) cuando se percibe el maniquí como un paciente propiciando el desempeño como enfermeros en un ambiente seguro en el cual se permite la repetición cuantas veces sea necesario para adquirir la habilidad.

En este sentido, en el año 2018 se diseñó y se construyó un RED para la enseñanza-aprendizaje de la inserción del catéter venoso periférico en estudiantes de enfermería. Este recurso determinó, posteriormente, un proceso investigativo que permitió responder la pregunta de cuál es la eficacia del RED "inserción del catéter venoso periférico" en el aprendizaje teóricoconceptual y procedimental en estudiantes de enfermería. Con los hallazgos, es posible incorporar una estrategia educativa efectiva probada mediante investigación en el currículo de formación. 


\section{MÉTODOS}

2.1. Diseño del estudio. Estudio cuasiexperimental desarrollado en la Facultad de Enfermería de la Universidad Nacional de Colombia con dos grupos de intervención (G1, G2) y un grupo control (GC). Los participantes del grupo G1 recibieron la intervención 1, los del grupo G2 recibieron la intervención 2 y los del grupo control recibieron la intervención tradicional.

2.2. Población, muestra y protocolo de muestreo. La muestra incluyó a 91 estudiantes asignados a tres grupos: 35 al grupo control (GC), 32 al grupo G1 y 24 al grupo G2. El muestreo se realizó a conveniencia debido a las características específicas de la población: estar inscrito en la primera asignatura del componente disciplinar de enfermería en la que inician su formación clínica y al número limitado de estudiantes matriculados en la asignatura. Se consideraron como criterios de inclusión: ser estudiante de enfermería que inicia la formación de cuidado básico y que no hubiera estado expuesto a la práctica clínica. Fueron excluidos aquellos con formación de auxiliar de enfermería o con experiencia en la ejecución del procedimiento. El estudio se desarrolló entre el año 2018 y 2019. Los participantes que cumplieron los criterios de inclusión y que aceptaron participar voluntariamente en el estudio firmaron el consentimiento informado.

2.3. Instrumentos de medición. Para la medición del conocimiento teórico- conceptual se aplicó una prueba de conocimiento y para la medición de la habilidad procedimental, una lista de chequeo.

- Prueba de conocimiento: fue diseñada por los autores como resultado de un proceso de revisión de literatura de la práctica de enfermería basada en evidencia (8-13), discusión frente a las experiencias tanto clínicas como docentes y a partir del contenido del RED. Incluyo 16 preguntas de selección múltiple con única respuesta clasificadas así: bioseguridad (preguntas 1,2 ), indicaciones de la venopunción (preguntas 3,4 ), contraindicaciones de la venopunción (preguntas 5,6), elección de la vena (preguntas 7, 8), equipos y elementos a utilizar en el procedimiento (preguntas 9,10), ejecución del procedimiento (preguntas11,12), complicaciones del procedimiento (preguntas 13,14) y seguimiento (preguntas 15,16). El puntaje total de la prueba fue de 5 puntos y todas las preguntas tenían el mismo peso porcentual. La aplicación de la prueba tomó entre 15 y 20 minutos, y fue aplicada por auxiliares de investigación.

- Lista de chequeo de habilidades procedimentales: fue diseñada por los autores como resultado de un proceso de revisión de literatura de la práctica de enfermería basada en evidencia (ㅇ-13), discusión frente a las experiencias tanto clínicas como docentes y a partir del contenido del RED. Incluyo 55 ítems para evaluar: preparación del paciente (4 ítems), alistamiento del equipo (11 ítems), purga del equipo (10 ítems) y procedimiento (30 ítems). Se calificó en forma dicotómica (0 para no ejecución; 1 para su ejecución). El puntaje total de la lista de chequeo fue de 5 puntos, con el mismo peso porcentual para cada ítem. Para la evaluación de las habilidades a partir de la lista de chequeo, los participantes fueron grabados en dos momentos. La ejecución y grabación del procedimiento de inserción del catéter venoso periférico tomó en promedio 20 minutos por participante y las videograbaciones fueron realizadas por camarógrafos entrenados, quienes no conocían a los participantes y pertenecían a otras disciplinas diferentes al área de la salud. Los investigadores realizaron una prueba piloto para estandarizar la evaluación de los videos mediante la lista de chequeo, 
corrigieron las desviaciones y unificaron los criterios de evaluación de los ítems. Cada video fue identificado con un código por auxiliares de investigación y posteriormente estos fueron evaluados por los investigadores.

2.4 Elaboración y contenido del recurso educativo digital (RED). Diseñado por los investigadores a partir de la reflexión pedagógica de cómo aprenden los estudiantes de enfermería este procedimiento y a partir de la revisión de literatura de la práctica de enfermería basada en evidencia, se elaboró el contenido del RED. Este contiene siete secciones: generalidades (que incluye definición, indicaciones, contraindicaciones, elección de la vena y bioseguridad), equipos e insumos (selección del catéter, selección del torniquete, selección del equipo de infusión, selección del antiséptico, fijación del catéter), procedimiento (que incluye videos demostrativos que contienen las diferentes fases del procedimiento, tales como preparación del paciente, alistamiento del equipo, purga del equipo y procedimiento de inserción de catéter venoso periférico para colocar líquidos intravenosos), seguimiento, complicaciones (flebitis, infiltración, embolismo), aspectos bioéticos y ejemplos de registros de enfermería, y bibliografía.

Las secciones del RED contenían material interactivo, imágenes, ilustraciones, animaciones y videos demostrativos realizados por los investigadores en el laboratorio de simulación, e incluía actividades interactivas de autoevaluación. EI RED estimuló los tipos de memoria (visual, auditiva, contextual), el pensamiento crítico y reflexivo.

2.5. Procedimiento. Se realizaron cinco mediciones y previo a estas todos los participantes estaban en igualdad de condiciones, inscritos por primera vez en la asignatura, no tenían conocimiento del procedimiento, ni formación clínica. La primera medición, consistió en una prueba de conocimiento (PC), aplicada a los tres grupos para medir el nivel de conocimiento teórico-conceptual antes del inicio de la intervención. La segunda (PC) se realizó al terminar la parte uno de la intervención. La medición tres $(P C)$ se realizó una semana después de la intervención parte 1. La cuarta medición (PC) se realizó al finalizar la intervención parte 2. Finalmente, la quinta (PC), se realizó dos semanas después de la intervención parte tres (Tabla 1).

Tabla 1. Procedimiento del estudio

\begin{tabular}{|c|c|c|c|c|c|c|c|c|}
\hline Grupo & $\begin{array}{c}\text { Medición } \\
\mathbf{1}\end{array}$ & $\begin{array}{c}\text { Intervención } \\
\text { parte 1 }\end{array}$ & $\begin{array}{c}\text { Medición } \\
\mathbf{2}\end{array}$ & $\begin{array}{c}\text { Medición } \\
\mathbf{3}\end{array}$ & $\begin{array}{c}\text { Intervención } \\
\text { parte }\end{array}$ & $\begin{array}{c}\text { Medición } \\
\mathbf{4}\end{array}$ & $\begin{array}{c}\text { Intervención } \\
\text { parte } \mathbf{3}\end{array}$ & $\begin{array}{c}\text { Medición } \\
\mathbf{5}\end{array}$ \\
\hline G1 & PC & CT1 & PC & PC & $\begin{array}{c}\text { LT1 } \\
\text { VG1 }\end{array}$ & $\begin{array}{c}\text { PC } \\
\text { LCh1 }\end{array}$ & $\begin{array}{c}\text { LRT1 VG2 } \\
\text { VG }\end{array}$ & $\begin{array}{c}\text { PC } \\
\text { LCh2. }\end{array}$ \\
\hline G2 & PC & CT1 & PC & PC & $\begin{array}{c}\text { LT2 } \\
\text { VG1 }\end{array}$ & $\begin{array}{c}\text { PC } \\
\text { LCh1 }\end{array}$ & $\begin{array}{c}\text { LRT2 } \\
\text { VG2 }\end{array}$ & $\begin{array}{c}\text { PC } \\
\text { LCh2 }\end{array}$ \\
\hline GC & PC & CT2 & PC & PC & $\begin{array}{c}\text { LT3 } \\
\text { VG1 }\end{array}$ & $\begin{array}{c}\text { PC } \\
\text { LCh1 }\end{array}$ & $\begin{array}{c}\text { LRT3 } \\
\text { VG2 }\end{array}$ & $\begin{array}{c}\text { PC } \\
\text { LCh2 }\end{array}$ \\
\hline
\end{tabular}

Variables evaluadas: PC: Prueba de conocimientos, LCh1: Lista de chequeo 1, LCh2: Lista de chequeo 2, VG1: Video grabación 1, VG2: Video grabación 2.

Intervenciones realizadas: CT1: Clase tipo 1, CT2: Clase tipo 2, LT1: Laboratorio tipo 1, VG1, LT2 Laboratorio tipo 2, VG1, LT3: Laboratorio tipo 3, VG1, LRT1: Laboratorio de refuerzo tipo 1, VG2, LRT2: Laboratorio de refuerzo tipo 2, VG2, LRT3: Laboratorio de refuerzo tipo 3, VG2.

Fuente: elaboración propia 


\section{Intervención G1}

Estuvo conformada por la CT1 (clase con profesor entrenado + RED), el LT1 (Laboratorio con RED + profesor entrenado), la VG1 (videograbación 1), el LRT1 (Laboratorio refuerzo con RED + profesor entrenado) y la VG2 (videograbación 2). Sobre la CT1: los estudiantes recibieron instrucción de navegación por las secciones del RED: definición, indicaciones, contraindicaciones, elección de la vena, bioseguridad, equipos, procedimiento y nota de enfermería. La instrucción se realizó en un aula equipada con computador para cada participante con una duración de 2 horas. El enlace del RED se dejó a partir de ese momento habilitado en la plataforma Moodle.

Para el LT1, los profesores entrenados en el RED proyectaron el video del procedimiento "inserción de catéter venoso periférico" tres veces durante 30 minutos. Posteriormente, cada estudiante realizó el procedimiento en simuladores part task trainers (brazos intravenosos para adultos preparados con líquido rojo para simular el retorno de sangre). Esta actividad se realizó en el laboratorio de simulación durante tres horas y participaron 32 estudiantes distribuidos en cuatro escenarios simulados de contexto hospitalario dotados con computador, televisor, camilla, simuladores y kits de insumos intravenosos. Para resolver las dudas de los participantes, el profesor entrenado se limitó a proyectar de nuevo el video para que el estudiante se orientara y ejecutara el procedimiento, posteriormente, se realizó la VG1.

Para el LRT1, se usó la misma metodología del LT1 durante los 15 días de la medición cero, los estudiantes fueron distribuidos en grupos de cuatro integrantes. El laboratorio estuvo a cargo de un docente de enfermería con experiencia clínica de 15 años, quien recibió entrenamiento por parte de los investigadores en el uso del RED para asegurar la fidelidad al protocolo experimental. Posteriormente se realizó la VG2.

\section{Intervención G2}

Estuvo conformada por la CT1 (clase con profesor entrenado + RED), el LT2 (laboratorio autónomo con RED), la VG1 (videograbación 1), el LRT2 (laboratorio de refuerzo autónomo con RED) y la VG2 (videograbación 2). La CT1 fue la misma del G1. En el LT2 auxiliares de investigación entrenados proyectaron el video del procedimiento "inserción de catéter venoso periférico" tres veces durante 30 minutos, luego los estudiantes autónomamente y sin supervisión, realizaron el procedimiento en los simuladores part task trainers. Este laboratorio se realizó durante 3 horas, con 24 participantes distribuidos en cuatro escenarios simulados de contexto hospitalario (con las mismas características del LT1). En el LRT2 se usó la misma metodología del LT2 a los 15 días de la medición cero. Posteriormente se realizó la VG2.

\section{Intervención GC o tradicional}

Esta intervención estuvo conformada por la CT2 (clase tradicional), el LT3 (laboratorio tradicional), la VG1 (videograbación 1), el LRT3 (laboratorio de refuerzo tradicional) y la VG2 (videograbación 2). La CT2 duró 2 horas y abordó varios temas, incluido el procedimiento de inserción de catéter venoso periférico, con metodología magistral y presentación en Power Point. Esta sesión estuvo a cargo de una profesora con trayectoria docente de ocho años en la temática. 
EI LT3 fue realizado por los profesores adscritos a la asignatura, quienes dieron una explicación general del procedimiento, hicieron la demostración y posteriormente algunos estudiantes lo ejecutaron en los simuladores part task trainers con el acompañamiento y corrección del docente, con una duración de tres horas. Los estudiantes fueron distribuidos en escenarios dotados con una mesa, los part task trainers de brazos intravenosos y el kit de insumos intravenosos. Posteriormente se realizó la VG1.

Finalmente, el LRT3 usó la misma metodología del LT3. Este se realizó a los 15 días de la medición cero, estuvo a cargo de una profesora asignada para actividades de refuerzo del laboratorio de la facultad. Posteriormente se realizó la VG2. Al final del estudio los participantes del GC, y los que no participaron en el estudio tuvieron acceso al RED disponible en la plataforma Moodle.

2.6. Análisis estadístico. Se analizaron los datos sociodemográficos con distribuciones de frecuencias. Para las variables de conocimiento teórico-conceptual y habilidad procedimental, se usaron pruebas de hipótesis con previa verificación de ajuste al modelo gaussiano con la prueba de normalidad de Kolmogorov Smirnov, con la cual se comprobó que no hubo ajuste. Para las comparaciones entre los grupos experimentales y de control, se utilizó la prueba de Kruskall Wallis. Para las comparaciones intragrupos, se utilizó la prueba de Friedman y Wilcoxon con rango signado para muestras relacionadas. Como nivel de significancia, se tomó un valor $\mathrm{p}$ menor a 0.05 . Los datos fueron procesados en el software SPSS versión 24 licenciado para la Universidad Nacional de Colombia.

2.7. Aspectos éticos. El estudio mediante el acta $N$ 11, fue aprobado por el Comité de ética en Investigación de la Facultad de Enfermería de la Universidad Nacional de Colombia. Los participantes fueron informados de los propósitos del estudio, su participación fue voluntaria, podrían retirarse en cualquier momento sin ningún tipo de repercusión académica.

\section{RESULTADOS}

A continuación se presentan los resultados obtenidos, iniciando con la descripcion de la muestra estudiada, seguido de las caracteristicas sociodemograficas, y los resultados comparativos intergrupos e intragrupos en el aprendizaje teórico, conceptual y procedimental de estudiantes de enfermería antes y despues de las intervenciones.

3.1. Descripción de la muestra estudiada. 91 estudiantes completaron el estudio, con 35 asignados en el grupo control (GC), 32 al grupo intervención 1 (G1) y 24 participantes al grupo intervención 2 (G2). La tasa de pérdida fue del 13\% (Gráfico 1). 


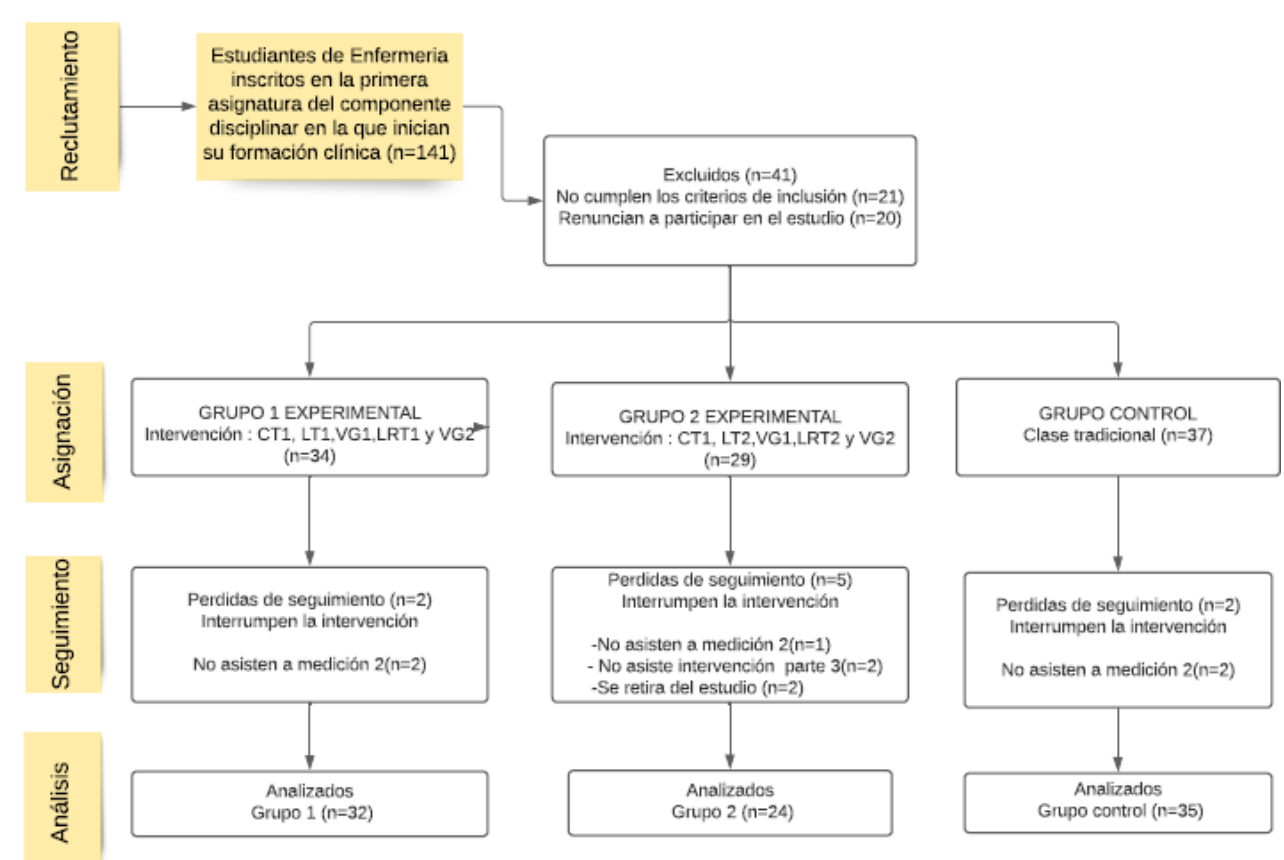

Gráfico 1. Diagrama del comportamiento de los participantes del estudio Fuente: Elaboración propia

\subsection{Características sociodemográficas}

El $62 \%$ de los participantes son mujeres, el $85 \%$ su edad oscilaba entre 17 y 20 años, seguidos por el grupo entre 21 a 24 años (13\%).

\subsection{Comparación intergrupos}

\subsubsection{Conocimiento teórico-conceptual}

Como se observa en la Tabla 2, en la medición 1 (preintervención parte 1) hay diferencias significativas entre los grupos $\mathrm{G} 1, \mathrm{G} 2$ y $\mathrm{GC}(\mathrm{p}=0.01)$. Las diferencias radicaron en que el grupo GC presentó un mejor desempeño en la prueba de conocimientos que los G1 y G2. Para la medición 2 (post intervención parte 1), hubo diferencias significativas entre los tres grupos $(p=0.01)$, observándose mejores puntuaciones en los grupos $\mathrm{G} 1$ y G2 que en el grupo GC.

Para la medición 3 (preintervención parte 2), se reportaron diferencias significativas en los tres grupos ( $p=0.01$ ) con incremento en los puntajes, aunque mejores en comparación para los $\mathrm{G} 1$ y G2 frente al grupo GC. En la medición 4 (posintervención parte 2), se observaron diferencias significativas en los tres grupos $(p=0.01)$ con mejores puntajes reportados para los $G 1$ y $G 2$ en comparación con el GC.

Finalmente, en la medición 5 (posintervención parte 3) se observaron diferencias significativas entre los grupos ( $p=0.01$ ), siendo los mejores puntajes para el $\mathrm{G} 1$ y el $\mathrm{G} 2$ en comparación con 
el GC, el cual continuó con puntajes similares a las mediciones previas. Como se observa, aunque el GC empezó el estudio en ventaja frente a los grupos G1 y G2, con el desarrollo de la intervención, los grupos G1 y G2 alcanzaron y superaron los puntajes del grupo GC.

Tabla 2. Conocimiento teórico-conceptual intergrupos

\begin{tabular}{cccccccc}
\hline Medición & $\begin{array}{c}\text { Grupos/conocimiento } \\
\text { teórico conceptual }\end{array}$ & Media & Mediana & DE & Min & Max $\begin{array}{c}\text { Kruskall } \\
\text { Wallis } \\
\text { Valor } \mathbf{p}\end{array}$ \\
\hline \multirow{3}{*}{1} & G1 & 1,8 & 1,9 & 0,75 & 0,62 & 3,8 & \\
& G2 & 2,3 & 2,2 & 0,63 & 1,3 & 3,4 & 0,01 \\
2 & GC & 2,6 & 2,5 & 0,54 & 1,6 & 3,8 & \\
& G1 & 3,5 & 3,8 & 0,47 & 2,5 & 4,4 & \\
& G2 & 3,6 & 3,8 & 0,62 & 2,5 & 5,0 & 0,01 \\
3 & GC & 2,8 & 2,8 & 0,47 & 1,8 & 3,8 & \\
& G1 & 3,7 & 3,8 & 0,57 & 2,5 & 4,7 & \\
4 & G2 & 3,5 & 3,6 & 0,8 & 0,62 & 4,4 & 0,01 \\
& GC & 3,1 & 3,1 & 0,47 & 2,1 & 4,1 & \\
& G1 & 3,7 & 3,8 & 0,81 & 0,00 & 4,7 & \\
5 & G2 & 3,7 & 3,8 & 0,47 & 2,8 & 4,7 & 0,01 \\
& GC & 3,0 & 3,1 & 0,53 & 1,9 & 3,8 & \\
& G1 & 4,0 & 4,1 & 0,46 & 3,1 & 5,0 & \\
& G2 & 3,7 & 3,8 & 0,58 & 2,5 & 4,7 & 0,01 \\
\hline
\end{tabular}

Fuente: Elaboración propia

\subsubsection{Habilidad procedimental}

Como se observa en la Tabla 3, en la medición 4 (posintervención parte 2) hay diferencias significativas entre los grupos $\mathrm{G} 1, \mathrm{G} 2$ y $\mathrm{GC}(\mathrm{p}=0.01)$. Las diferencias radicaron en que el grupo G1 presentó mejores puntajes en comparación con los grupos G2 y GC. En la medición 5 (posintervención parte 3 ), se observaron diferencias significativas entre los grupos $(p=0.01)$, siendo los mejores puntajes para el G1 y seguido del grupo G2. El GC, aunque presentó un ligero incremento en los puntajes, no superó ni al G1 ni al G2.

Tabla 3. Habilidad procedimental intergrupos

\begin{tabular}{lccccccc}
\hline $\begin{array}{l}\text { Medición } \\
\text { /video }\end{array}$ & $\begin{array}{c}\text { Grupos/habilidad } \\
\text { procedimental }\end{array}$ & Media & Mediana & DE & Min & Max $\begin{array}{c}\text { Kruskall } \\
\text { Wallis } \\
\text { Valor } \mathbf{p}\end{array}$ \\
\hline \multirow{2}{*}{1 (VG1) } & G1 & 4,0 & 4,0 & 0,42 & 2,7 & 4,8 & \\
& G2 & 3,5 & 3,5 & 0,49 & 2,6 & 4,7 & 0,01 \\
& GC & 2,4 & 2,4 & 0,48 & 1,5 & 3,4 & \\
$2($ VG2) & G1 & 4,4 & 4,5 & 0,43 & 3,5 & 5,0 & \\
& G2 & 3,9 & 4,0 & 0,59 & 2,6 & 4,7 & 0,01 \\
\hline
\end{tabular}

Fuente: Elaboración propia 


\subsection{Comparación intragrupos}

\subsubsection{Conocimiento teórico-conceptual}

Se observa en la Tabla 4 cómo para las cinco mediciones realizadas el comportamiento al interior de cada grupo tuvo diferencias significativas $(p=0.01)$. Sin embargo, al observar las medianas de los G1 y G2, es claro que el aumento en el puntaje al interior de los grupos fue mayor, en comparación con el aumento del puntaje del GC. Frente a este último, se puede afirmar que la intervención tradicional causó un efecto positivo en el aumento del conocimiento teórico conceptual; sin embargo, este aumento fue mayor en los grupos que recibieron la intervención diseñada para este estudio. Frente a los grupos de intervención, es llamativo que el desempeño del G1 superó al del G2, lo que muestra que el RED por sí solo es útil y eficaz para mejorar el conocimiento teórico conceptual; no obstante, el RED con el acompañamiento de un recurso humano entrenado resulta significativamente mejor.

Tabla 4. Conocimiento teórico-conceptual Intragrupos

\begin{tabular}{cccccccc}
\hline Grupo & Medición & Media & Mediana & DE & Min & Max & $\begin{array}{c}\text { Prueba } \\
\text { Wilcoxon } \\
\text { Valor } \mathbf{p}\end{array}$ \\
\hline \multirow{4}{*}{ G1 } & 1 & 1,8 & 1,9 & 0,75 & 0,62 & 3,8 & \\
& 2 & 3,5 & 3,8 & 0,47 & 2,5 & 4,4 & \\
& 3 & 3,7 & 3,8 & 0,57 & 2,5 & 4,7 & 0,01 \\
& 4 & 3,7 & 3,8 & 0,81 & 0,00 & 4,7 & \\
G2 & 5 & 4,0 & 4,1 & 0,46 & 3,1 & 5,0 & \\
& 1 & 2,3 & 2,2 & 0,63 & 1,3 & 3,4 & \\
& 2 & 3,6 & 3,8 & 0,62 & 2,5 & 5,0 & \\
& 3 & 3,5 & 3,6 & 0,79 & 0,62 & 4,4 & 0,01 \\
& 4 & 3,8 & 3,8 & 0,47 & 2,8 & 4,7 & \\
GC & 5 & 3,7 & 3,8 & 0,58 & 2,5 & 4,7 & \\
& 1 & 2,6 & 2,5 & 0,54 & 1,6 & 3,8 & \\
& 2 & 2,8 & 2,8 & 0,47 & 1,9 & 3,8 & \\
& 3 & 3,1 & 3,1 & 0,47 & 2,1 & 4,1 & 0,01 \\
& 4 & 3,0 & 3,1 & 0,53 & 1,9 & 3,8 & \\
\hline
\end{tabular}

Fuente: Elaboración propia

\subsubsection{Habilidad procedimental}

En la Tabla 5 se observa que, para las dos mediciones realizadas el comportamiento al interior de cada grupo tuvo diferencias significativas $(p=0.01$ y $p=0.03$ ). Sin embargo, al observar las medianas de los grupos G1 y G2 es claro que estos grupos iniciaron en ventaja con respecto al GC y, con el desarrollo de la intervención, los tres grupos superaron los puntajes iniciales, observándose mejores puntajes en los G1 y G2. Del GC, se puede afirmar que la intervención tradicional causó un efecto positivo en el aumento de la habilidad procedimental; este aumento fue mayor en los grupos que recibieron la intervención diseñada para este estudio. Frente a los grupos de intervención, es llamativo que el desempeño del G1 superó al del G2, lo que muestra que el RED por sí solo es útil y eficaz para mejorar la habilidad procedimental. Sin embargo, el RED acompañado de un recurso humano entrenado y la repetición de la habilidad procedimental, resulta significativamente mejor. 
Tabla 5. Habilidad procedimental intragrupos después de las intervenciones

\begin{tabular}{cccccccc}
\hline Grupo & Medición/video & Media & Mediana & DE & Min & Max & $\begin{array}{c}\text { Prueba } \\
\text { Wilcoxon } \\
\text { Valor } \mathbf{p}\end{array}$ \\
\hline \multirow{2}{*}{ G1 } & 1 & 4,0 & 4,0 & 0,42 & 2,7 & 4,8 & 0,01 \\
& 2 & 4,4 & 4,5 & 0,43 & 3,5 & 5,0 & \\
G2 & 1 & 3,5 & 3,5 & 0,5 & 2,6 & 4,7 & 0,03 \\
& 2 & 3,9 & 4,0 & 0,6 & 2,6 & 4,7 & \\
GC & 1 & 2,4 & 2,4 & 0,5 & 1,5 & 3,4 & 0,01 \\
& 2 & 3,0 & 3,2 & 0,68 & 0,6 & 3,9 & 0,0 \\
\hline
\end{tabular}

Fuente: Elaboración propia

\section{DISCUSIÓN}

Evaluar la eficacia de un RED en el aprendizaje teórico-conceptual y procedimental de la inserción del catéter venoso periférico en estudiantes de enfermería, es importante para diseñar e incorporar estrategias educativas efectivas probadas mediante investigación en los currículos de formación para enfermeros. Los nuevos desafíos en la educación de las ciencias de la salud y en la seguridad de los pacientes exigen el desarrollo y la implementación de estrategias de enseñanza alternativas como la que se propone en este estudio. Por ello, conocer la eficacia de esta estrategia en el aprendizaje es relevante para la toma de decisiones en el proceso de formación de estudiantes de enfermería.

Los hallazgos muestran un efecto positivo del RED en el aprendizaje teórico-conceptual y de habilidad procedimental para la inserción del catéter venoso periférico en estudiantes de enfermería, con diferencias significativas en los tres grupos. En este sentido, se observa un mejor desempeño en los grupos que recibieron la intervención, lo que demuestra la efectividad del RED por sí solo, aunque, cuando es acompañado de un recurso humano entrenado es significativamente mejor.

El comportamiento de los grupos experimentales mostró diferencias significativas en el conocimiento teórico-conceptual posterior a las intervenciones. Aunque el GC inició en ventaja frente a los G1 y G2, este tuvo un leve incremento, pero no superó los grupos experimentales. La enseñanza tradicional causó un efecto positivo en el aumento de la habilidad procedimental en el GC; sin embargo, el desempeño de los grupos experimentales con el uso del RED fue superior.

Estos resultados pueden tener como explicación la construcción del RED a partir de una reflexión pedagógica sobre cómo se aprenden los procedimientos clínicos, reflexión que tuvo en cuenta conceptos de e-learning y b-learning para facilitar el conocimiento en los estudiantes (14), de modo que pudiera permitir una mejor aproximación desde el contexto teórico a la práctica. En este sentido, se destaca que los RED favorecen el cambio en modelos de enseñanza centrados en lo que el profesor enseña en el aula (input) hacia aquellos fundamentados en el estudiante y su aprendizaje (output) (15).

EI RED que se utilizó estimuló en los estudiantes los distintos tipos de memoria, dado que incluyó material interactivo, imágenes, ilustraciones, animaciones y videos demostrativos que facilitaron una aproximación auditiva, visual y contextual al proporcionar realismo de cada 
experiencia, elementos que fueron tenidos en cuenta en el diseño de la didáctica incorporada. Así, se constituye en una estrategia innovadora que incorpora aspectos que facilitan el autoaprendizaje, la autogestión, la autonomía y optimiza el tiempo en el encuentro entre el profesor y el estudiante, a la par que permite una enseñanza inclusiva y equitativa, ya que los elementos teóricos y prácticos están contenidos en un solo recurso disponible para su revisión permanente por parte de los participantes en igualdad de condiciones (16).

Los resultados en el aprendizaje son superiores cuando el docente utiliza recursos educativos de este tipo y se adhiere al contenido para estandarizar la enseñanza en los procedimientos clínicos, dado que los contenidos se basan en lo reportado en la literatura científica, lo que evita la enseñanza de conceptos y prácticas inadecuadas sin fundamento teórico actualizado. Así mismo, el docente puede dar respuesta a dudas e inquietudes que surgen en el proceso de formación, lo que fortalece, mediante la interacción docente-estudiante, el desarrollo de habilidades no técnicas fundamentales en los profesionales de enfermería.

La generación actual de los estudiantes en la educación superior requiere de estrategias innovadoras para el aprendizaje diferentes a las empleadas tradicionalmente, las cuales se han caracterizado por estar centradas en el profesor y en el aula, lo que genera desmotivación y bloquea el aprendizaje (17). Por ello, el aprendizaje de procedimientos clínicos puede optimizarse con la combinación de los recursos educativos a través de la web y la práctica en simulación clínica, porque en forma simultánea se puede observar el procedimiento en los videos del RED, abordar a la persona y ejecutar las habilidades psicomotoras en un contexto de cuidado simulado previo al contacto con el paciente real. En vista de lo anterior, la simulación clínica es un proceso de aprendizaje experiencial que proporciona un entorno seguro de aprendizaje, promueve el análisis, la reflexión, el pensamiento crítico, la repetición por ensayo y error (18), genera empoderamiento, seguridad y confianza en el estudiante (19).

La enseñanza de habilidades prácticas requiere de varios métodos en conjunto con la revisión tradicional de los conceptos. Sin embargo, Doijad (20) evidencia en estudiantes de fisiología que los videos pueden llegar a ser una sustitución a la demostración en tiempo real cuando esta no está disponible. En este estudio, se observó un incremento en la habilidad procedimental en los grupos experimentales, lo que demuestra la efectividad del RED para el aprendizaje de un procedimiento clínico. Estos hallazgos resultan contrarios a lo encontrado en el estudio de Devi (21), quien comparó la efectividad de un programa de enseñanza asistido por video frente a la demostración tradicional en el aprendizaje de habilidades de palpación obstétrica en estudiantes de enfermería. En tal investigación, no se encontró diferencia significativa entre ambas estrategias, aunque el promedio general de las habilidades en la posprueba fue mayor en el grupo expuesto frente a la demostración tradicional (21).

No obstante, al igual que en el estudio de Kaveevivitchai (donde se evaluó la efectividad del aprendizaje mediado por multimedia en la evaluación de los signos vitales en estudiantes de enfermería en Tailandia) (22), en la presente investigación las prepruebas para medir el conocimiento previo a la intervención en los tres grupos fueron significativamente diferentes de las pospruebas; igualmente los puntajes (promedio) en la lista de verificación en ambos estudios fueron estadísticamente diferentes comparados con el grupo tradicional del trabajo de Tailandia y del grupo control del presente trabajo. El desempeño fue mejor en los dos grupos expuestos al recurso que se quería medir en ambos estudios frente al grupo sometido a la enseñanza tradicional. Así, la simulación es más un complemento de la práctica clínica que un sustituto (203). 
En otro estudio, Bloomfield et al. (24) no encontraron diferencia significativa en el aprendizaje del lavado de manos en estudiantes de enfermería (en conocimiento y habilidad) al comparar la enseñanza entre un método convencional en el aula y un método de aprendizaje multimedia e interactivo asistido por computadora. Las mediciones del conocimiento reportaron incremento desde el inicio, pero no hallaron diferencia significativa entre los dos grupos. El rendimiento en el aprendizaje de la habilidad del lavado de manos a las dos semanas fue similar en los dos grupos, pero con una diferencia significativa a las ocho semanas a favor del grupo de intervención.

Por su parte, el estudio de Morente et al. (25) evaluó la efectividad de una herramienta de elearning en el aprendizaje teórico-conceptual de la evaluación de úlceras por presión en estudiantes al compararla con el método de enseñanza tradicional medida a través de preprueba y posprueba. Así, encontraron mayor efectividad en el aprendizaje mediado por elearning ( $p=0.01)$, aunque se tuvo un tamaño pequeño de muestra.

Asimismo, Garner et al. (26) midieron la efectividad de una intervención educativa (mediante simulación de baja fidelidad) sobre el conocimiento y la habilidad para la obtención de un acceso venoso periférico en 180 enfermeras, a través de preprueba, posprueba y listas de verificación. Los hallazgos mostraron una mejora estadísticamente significativa $(p=0.01)$, en otras palabras, el $95 \%$ de las enfermeras participantes del estudio mejoraron sus conocimientos y habilidades posterior a la intervención educativa. Cabe anotar que dicha estrategia es útil en países de medianos y bajos ingresos o en otros entornos en donde la experiencia práctica es limitada en los programas de educación en enfermería

No obstante, el aprendizaje basado en estrategias de e-learning tiene limitaciones como la naturaleza impersonal (27) y en algunas circunstancias los aspectos relacionados con la accesibilidad. Sin embargo, cuando el e-learning se combina con métodos de enseñanza cara a cara entre el docente y el estudiante, el aprendizaje se potencializa (28). De igual forma, cuando se combinan métodos innovadores en la educación de enfermería se incrementa la motivación, se facilita el aprendizaje y se mejoran las habilidades de los estudiantes, garantizando así la seguridad del paciente y la calidad del cuidado en el futuro (29).

Los resultados de este estudio se constituyen en un importante insumo para la docencia en enfermería, que avanza hacia un enfoque constructivista que requiere la incorporación de nuevas estrategias didácticas a partir del uso de las tecnologías de la información y las comunicaciones (TIC) ( $\underline{30})$, capaces de responder a los desafíos actuales de la práctica. Al evidenciarse la eficacia del RED utilizado, este podría implementarse en los entornos de simulación para promover el aprendizaje autónomo en el estudiante, quien podría ejecutar la habilidad en múltiples oportunidades por sí solo con el apoyo del RED, incrementando su curva de aprendizaje psicomotora y optimizando los tiempos de encuentro docente-estudiante.

En suma, en entornos simulados, el RED se convierte en un excelente complemento para practicar la habilidad psicomotora, porque promueve la repetición por ensayo y error, y es asequible para utilizar en contextos con recursos limitados (26). Del mismo modo, este recurso incrementa el conocimiento y es una herramienta útil de apoyo tanto para los profesores como para el desarrollo de los estudiantes en enfermería (31).

El estudio presenta algunas limitaciones. La falta de aleatorización probablemente incidió en las diferencias obtenidas en la primera medición entre los grupos, de igual forma este resultado se podría relacionar con las características propias de cada estudiante y por su aprendizaje 
autónomo previo a la clase, el muestreo a conveniencia permitió evitar contaminación entre los tres grupos dado que cursaban la misma asignatura, lo cual limita la generalización de los resultados.

Para futuros estudios se considera utilizar este protocolo de intervención, así como realizar la validez facial y de contenido a las listas de chequeo, con poblaciones mayoritarias realizar la aleatorización de los participantes, usar métodos mixtos que generen otros hallazgos y complementen la información del fenómeno y se generen otros aportes que favorezcan los procesos educativos. El RED puede tener mayor utilidad en la enseñanza, cuando este se incluye en el plan curricular institucional y los participantes del proceso enseñanza aprendizaje se adhieren a su contenido. Por otro lado, se puede comprobar la eficacia del RED en otros contextos de formación en enfermería y en educación continua a profesionales.

\section{CONCLUSIONES}

Los hallazgos indican que el RED “inserción del catéter venoso periférico” es eficaz por sí solo y significativamente mejor cuando se usa con el acompañamiento de un recurso humano entrenado durante el proceso de enseñanza-aprendizaje de la inserción del catéter venoso periférico en estudiantes de enfermería al incrementar el conocimiento teórico-conceptual y de habilidad procedimental $(\mathrm{p}=0.01)$.

En la primera medición el GC inició en ventaja frente a los grupos experimentales en el conocimiento teórico-conceptual, sin embargo, estos grupos alcanzaron al GC y lo superaron en los puntajes, lo que podría indicar un mayor efecto del RED en el aprendizaje. En relación con la habilidad procedimental, los grupos experimentales iniciaron con mejores resultados que el GC, los cuales se incrementaron al finalizar la intervención con diferencias estadísticamente significativas. La intervención tradicional tuvo un efecto positivo; sin embargo, no superó los grupos que recibieron las intervenciones diseñadas para este estudio.

Los resultados del estudio pueden estar relacionados con la motivación de los investigadores para trascender a pedagogías socio-constructivistas mediadas por las TIC a través del RED "inserción de catéter venoso periférico", elaborado a partir de la experiencia docente, de la reflexión pedagógica y de la revisión de la literatura para estimular los diferentes estilos de aprendizaje, generar autonomía y autogestión en el estudiante. Así mismo, los hallazgos pueden estar influidos por la concordancia entre el RED y la adherencia del docente al contenido que estandariza la enseñanza y por la posibilidad que tiene el RED para ser visualizado las veces que sea necesario mientras el estudiante ejecuta la inserción del catéter venoso con simuladores.

Así, futuros estudios podrían explorar la adherencia de los estudiantes a los contenidos del RED al finalizar la formación del pregrado, evaluar la transferencia del conocimiento obtenido del RED al entorno clínico y así mismo continuar con procesos investigativos para la construcción de recursos similares que faciliten la enseñanza-aprendizaje de los procedimientos clínicos en enfermería.

Contribución de los autores/Author Contributions: "Conceptualización, J.C.D.A., y L.N.R.A; adquisición de los fondos, J.C.D.A., y X.S.M; investigación, C.O.S., X.S.M., J.C.D.A., L.N.R.A; metodología, X.S.M., y C.O.S.; administración del proyecto, L.N.R.A recursos L.N.R.A; supervisión, C.O.S., X.S.M., L.N.R.A; validación, C.O.S.,y X.S.M; visualización, 
X.S.M., y C.O.S.; escritura: preparación del borrador original, C.O.S.,y X.S.M; escritura: revisión y edición, X.S.M., y C.O.S; Todos los autores han leído y aceptado la versión publicada del manuscrito".

Fondos: Esta investigación fue financiada por la Convocatoria Investigación sobre innovación pedagógica de la Universidad Nacional de Colombia, Sede Bogotá, 2017.

Conflicto de intereses: Los autores declaran no tener ningún conflicto de intereses. "Los financiadores no tuvieron ningún rol en el diseño del estudio; en la recopilación, análisis o interpretación de datos; en la redacción del manuscrito o en la decisión de publicar los resultados".

\section{REFERENCIAS}

1. Vizcarra C, Cassutt C, Corbitt N, Richardson D, Runde D, Stafford K. Recommendations for improving safety practices with short peripheral catheters. J Infus Nurs [Internet]. 2014 [citado el 5 de agosto de 2021];37(2):121-4. DOI: 10.1097/NAN.0000000000000028

2. De Souza-Junior VD, Mendes IAC, Marchi-Alves LM, Jackman D, Wilson-Keates B, De Godoy S. Peripheral Venipuncture Education Strategies for Nursing Students: An Integrative Literature Review. J Infus Nurs [Internet]. 2020 [citado el 5 de agosto de 2021];43(1):24-32. DOl: 10.1097/NAN.0000000000000351

3. Danski MT, Johann DA, Vayego SA, Oliveira GN, Lind J. Complicações relacionadas ao uso do cateter venoso periférico: ensaio clínico randomizado. Acta Paul Enferm [Internet]. 2020 [citado el 5 de agosto de 2021]; 29(1): 84-92. DOI: 10.1590/1982-0194201600012

4. Keleekai NL, Schuster CA, Murray CL, King MA, Stahl BR, Labrozzi LJ, et al. Improving Nurses' Peripheral Intravenous Catheter Insertion Knowledge, Confidence, and Skills Using a Simulation-Based Blended Learning Program: A Randomized Trial. Simul Healthc [Internet]. 2016 [citado el 5 de agosto de 2021];11(6):376-84. DOI: 10.1097/SIH.0000000000000186

5. Piñas Morales MB, Ávalos Pérez MA, Ávalos Pérez MC. Saber y aprendizaje con Tecnologías de la Información y la Comunicación (TIC), en la Educación Superior. Dominio de las Cienc. 2017;3(2):936-46. DOI: 10.23857/dc.v3i2.518

6. UNESCO. Enfoques estratégicos sobre las TICS en educación en América Latina y el Caribe [Internet]. 2013 [citado el 8 de diciembre de 2020]. p. 62. Disponible en: http://www.unesco.org/new/fileadmin/MULTIMEDIA/FIELD/Santiago/images/ticsesp.pdf

7. Handeland JA, Prinz A, Ekra EMR, Fossum M. The role of manikins in nursing students' learning: A systematic review and thematic metasynthesis. Nurse Educ Today [Internet]. 2021 [citado el 5 de agosto de 2021];98:104661. DOI: 10.1016/j.nedt.2020.104661

8. Asociación Profesional de Enfermeras de Ontario. Guía de buenas practicas en enfermeria. Cuidados y mantenimiento de los accesos vasculares para reducir las complicaciones [Internet]. 2008 [citado el 5 de agosto de 2021]. https://rnao.ca/sites/rnaoca/files/2014 CuidadoAccesoVascular spp 022014.pdf

9. Gorski LA. The 2016 Infusion Therapy Standards of Practice. Home Healthc Now [Internet]. 2017 [citado el 5 de agosto de 2021];35(1):10-8. DOl: 10.1097/NHH.0000000000000481

10. Achury D, Achury LF, Rodriguez SM, Diaz JC. Fundamentos enfermeros en el proceso de administración de medicamentos. 2012. Editorial Pontificia Universidad Javeriana. 2012.

11. Ansel B, Boyce M, Embree JL. Extending Short Peripheral Catheter Dwell Time: A Best Practice Discussion. J Infus Nurs [Internet]. 2017 [citado el 5 de agosto de 2021];40(3):143-46. DOl: 10.1097/NAN.0000000000000137 
12. Gorski LA, Hallock D, Kuehn SC, Morris P, Russell JM, Skala LC. Recommendations for frequency of assessment of the short peripheral catheter site. J Infus Nurs [Internet]. 2012 [citado el 5 de agosto de 2021];35(5):290-2. DOI: 10.1097/NAN.0b013e318267f636

13. Goudet V, Timsit JF, Lucet JC, Lepape A, Balayn D, Seguin S, Mimoz O. Comparison of four skin preparation strategies to prevent catheter-related infection in intensive care unit (CLEAN trial): a study protocol for a randomized controlled trial. Trials [Internet]. 2013 [citado el 5 de agosto de 2021];14:114. DOl: 10.1186/1745-6215-14-114

14. Parra Herrera LA. Blended leargning: La nueva formación en educación superior. Av Investig en Ing [Internet]. 2008 [citado el 8 de diciembre de 2020];1(9):95-102. https://revistas.unilibre.edu.co/index.php/avances/article/view/2653

15. Agencia Nacional de Evaluación de la Calidad y Acreditación - ANECA. Guía de apoyo para la redacción, puesta en práctica y evaluación de los resultados del aprendizaje [Internet]. 2014 [citado el 8 de diciembre de 2020]. p. 68. http://www.aneca.es/Documentos-ypublicaciones/Otras-guias-y-documentos-de-evaluacion/Guia-de-apoyo-para-la-redaccionpuesta-en-practica-y-evaluacion-de-los-RESULTADOS-DEL-APRENDIZAJE

16. Persky AM, Kirwin JL, Marasco CJ, May DB, Skomo ML, Kennedy KB. Classroom attendance: Factors and perceptions of students and faculty in US schools of pharmacy. Curr Pharm Teach Learn [Internet]. 2014 [citado el 8 de diciembre de 2020];6(1):1-9. DOI: 10.1016/..cptl.2013.09.014

17. Amandu GM, Muliira JK, Fronda DC. Using Moodle E-learning Platform to Foster Student Selfdirected Learning: Experiences with Utilization of the Software in Undergraduate Nursing Courses in a Middle Eastern University. Procedia - Soc Behav Sci [Internet]. 2013 [citado el 8 de diciembre de 2020];93:677-83. DOl: 10.1016/j.sbspro.2013.09.260

18. Schön DA. La formación de profesionales reflexivos: hacia un nuevo diseño de la enseñanza y el aprendizaje en las profesiones. Barcelona: Ediciones Paidós; 1992.

19. Hanshaw SL, Dickerson SS. High fidelity simulation evaluation studies in nursing education: $A$ review of the literature. Nurse Educ $\operatorname{Pr}$ [Internet]. 2020 [citado el 8 de diciembre de 2020];46:102818. DOI: 10.1016/i.nepr.2020.102818

20. Doijad V, Kamble P. A comparative study: Learning in Experimental Physiology with use of live animals V/S video demonstration as learning aids in first MBBS students. Int $\mathrm{J}$ Healthc Biomed Res [Internet]. 2013 [citado el 8 de diciembre de 2020];2(1):24-9. http://ijhbr.com/pdf/24-29.pdf

21. Devi B, Khandelwal B, Das M. Comparison of the effectiveness of video-assisted teaching program and traditional demonstration on nursing students learning skills of performing obstetrical palpation. Iran J Nurs Midwifery Res [Internet]. 2019 [citado el 8 de diciembre de 2020];24(2):118-23. https://www.ncbi.nlm.nih.gov/pmc/articles/PMC6390442/

22. Kaveevivitchai C, Chuengkriankrai B, Luecha Y, Thanooruk R, Panijpan B, Ruenwongsa P. Enhancing nursing students' skills in vital signs assessment by using multimedia computerassisted learning with integrated content of anatomy and physiology. Nurse Educ Today [Internet]. 2009 [citado el 8 de diciembre de 2020];29(1):65-72. DOl: 10.1016/i.nedt.2008.06.010

23. Bogossian FE, Cooper SJ, Cant R, Porter J, Forbes H. A trial of e-simulation of sudden patient deterioration (FIRST2ACT WEBTM) on student learning. Nurse Educ Today [Internet]. 2015 [citado el 8 de diciembre de 2020];35(10):e36-42. DOI: 10.1016/].nedt.2015.08.003

24. Bloomfield J, Roberts J, While A. The effect of computer-assisted learning versus conventional teaching methods on the acquisition and retention of handwashing theory and skills in prequalification nursing students: A randomised controlled trial. Int J Nurs Stud [Internet]. 2010 [citado el 8 de diciembre de 2020];47(3):287-94. DOI: 10.1016/i.ijnurstu.2009.08.003

25. Morente L, Morales-Asencio JM, Veredas FJ. Effectiveness of an e-learning tool for education on pressure ulcer evaluation. J Clin Nurs [Internet]. 2014 [citado el 8 de diciembre de 2020];23(13-14):2043-52. DOl: 10.1111/jocn.12450 
26. Garner SL, Prater LS, Raj L, Leena GV, Anitha AJJ. Effectiveness of Peripheral Intravenous Skill Continuing Education Using Low-Fidelity Simulation Among Nurses in India. J Contin Educ Nurs [Internet]. 2018 [citado el 8 de diciembre de 2020];49(6):255-61. DOI: 10.3928/0022012420180517-05

27. Korhonen T, Lammintakanen J. Web-based learning in professional development: experiences of Finnish nurse managers. J Nurs Manag [Internet]. 2005 [citado el 10 de diciembre de 2020];13(6):500-7. DOI: 10.1111/j.1365-2934.2005.00556.x

28. McDonald EW, Boulton JL, Davis JL. E-learning and nursing assessment skills and knowledge - An integrative review. Nurse Educ Today [Internet]. 2018 [citado el 8 de diciembre de 2020];66:166-74. DOI: 10.1016/j.nedt.2018.03.011

29. Culha I. Active learning methods used in nursing education. J Pedagog Res [Internet]. 2019 [citado el 8 de diciembre de 2020];3(2):74-86. DOI: 10.33902/JPR.2019254174

30. Padilha JM, Ribeiro A, Rosa J, Marques D, Machado PP. Clinical Virtual Simulation as Lifelong Learning Strategy_Nurse's Verdict. Clin Simul Nurs [Internet]. 2020 [citado el 8 de diciembre de 2020];47:1-5. DOI: 10.1016/i.ecns.2020.06.012

31. Frota NM, Galindo Neto NM, Barros LM, Pereira FGF, Melo GAA, Caetano JÁ. Hypermedia on peripheral venipuncture: effectiveness in teaching nursing students. Rev Bras Enferm [Internet]. 2018 [citado el 8 de diciembre de 2020];71(6):2983-9. DOI: 10.1590/0034-7167-2017-0205 